\title{
Mismatch Negativity Encoding of Prediction Errors Predicts S-ketamine-Induced Cognitive Impairments
}

\author{
André Schmidt*,', Rosilla Bachmann', Michael Kometer', Philipp A Csomor', Klaas E Stephan ${ }^{2,3}$, \\ Erich Seifritz ${ }^{4}$ and Franz $X$ Vollenweider ${ }^{1}$ \\ 'Neuropsychopharmacology and Brain Imaging, University Hospital of Psychiatry, University of Zurich, Zurich, Switzerland; '2 Laboratory for Social \\ and Neural Systems Research, Department of Economics, University of Zurich, Zurich, Switzerland; ${ }^{3}$ Wellcome Department of Neuroimaging, \\ University College London, London, UK; ${ }^{4}$ Clinic of Affective Disorders and General Psychiatry, University Hospital of Psychiatry, Zurich, Switzerland
}

\begin{abstract}
Psychotomimetics like the $\mathrm{N}$-methyl-D-aspartate receptor (NMDAR) antagonist ketamine and the 5-hydroxytryptamine2A receptor $\left(5-\mathrm{HT}_{2 \mathrm{~A}} \mathrm{R}\right)$ agonist psilocybin induce psychotic symptoms in healthy volunteers that resemble those of schizophrenia. Recent theories of psychosis posit that aberrant encoding of prediction errors (PE) may underlie the expression of psychotic symptoms. This study used a roving mismatch negativity ( $M M N$ ) paradigm to investigate whether the encoding of $\mathrm{PE}$ is affected by pharmacological manipulation of NMDAR or $5-\mathrm{HT}_{2 A} \mathrm{R}$, and whether the encoding of PE under placebo can be used to predict drug-induced symptoms. Using a doubleblind within-subject placebo-controlled design, S-ketamine and psilocybin, respectively, were administrated to two groups of healthy subjects. Psychological alterations were assessed using a revised version of the Altered States of Consciousness (ASC-R) questionnaire. As an index of PE, we computed changes in MMN amplitudes as a function of the number of preceding standards (MMN memory trace effect) during a roving paradigm. S-ketamine, but not psilocybin, disrupted PE processing as expressed by a frontally disrupted MMN memory trace effect. Although both drugs produced positive-like symptoms, the extent of PE processing under placebo only correlated significantly with the severity of cognitive impairments induced by S-ketamine. Our results suggest that the NMDAR, but not the $5-H_{2 A} R$ system, is implicated in PE processing during the MMN paradigm, and that aberrant PE signaling may contribute to the formation of cognitive impairments. The assessment of the MMN memory trace in schizophrenia may allow detecting early phases of the illness and might also serve to assess the efficacy of novel pharmacological treatments, in particular of cognitive impairments. Neuropsychopharmacology (2012) 37, 865-875; doi:I0.1038/npp.201 I.26I; published online 26 October 201 I
\end{abstract}

Keywords: model psychosis; NMDAR; 5-HT; predictive coding; MMN memory trace effect; cognitive impairment

\section{INTRODUCTION}

Dissociative $N$-methyl-D-aspartate receptor (NMDAR) antagonists such as ketamine and classic hallucinogens like psilocybin, which activate 5-hydroxytryptamine2A receptors $\left(5-\mathrm{HT}_{2 \mathrm{~A}} \mathrm{R}\right)$, constitute two models of psychosis that are used to study the neurobiology of psychotic symptom formation in schizophrenia (González-Maeso and Sealfon, 2009; Javitt and Zukin, 1991; Vollenweider and Geyer, 2001). Although administration of ketamine to healthy volunteers reproduces both positive and negative symptoms of schizophrenia (Adler et al, 1999; Malhotra et al, 1996; Vollenweider et al, 1997a), psilocybin engenders positive symptoms that are highly reminiscent of those observed in

\footnotetext{
*Correspondence: A Schmidt, Neuropsychopharmacology and Brain Imaging, University Hospital of Psychiatry Zurich, University of Zurich, Lenggstrasse 31, Zurich 8029, Switzerland, Tel: +4I 44384 2616, Fax: + 4 I 44384 2499, E-mail: andre.schmidt@bli.uzh.ch Received 18 May 2011; revised 27 September 2011; accepted 28 September 2011
}

schizophrenia (Geyer and Vollenweider, 2008; GouzoulisMayfrank et al, 1998). In neuroimaging studies, both ketamine and psilocybin altered the activity in frontotemporal regions associated with depersonalization and thought disorder (Vollenweider et al, 1997a, b). Furthermore, ketamine infusion in schizophrenia patients transiently exacerbated positive symptoms (Lahti et al, 1995b) associated with an activation of PFC and thalamic structures (Lahti et al, 1995a). Although ketamine- and psilocybin-like drugs differ in their primary mechanism of action, both classes of drugs increase extracellular glutamate in PFC (Moghaddam et al, 1997; Muschamp et al, 2004), which may explain some of the common psychotic symptoms. However, while the pharmacological properties of these psychotomimetics are well documented (Fantegrossi et al, 2008; Nichols, 2004), the neuronal and cognitive mechanisms underlying ketamine- and psilocybin-induced symptoms are less well understood.

Recent works suggest that the formation of symptoms induced by psychomimetics could be understood within the framework of predictive coding models (Corlett et al, 
2009, 2011). These suggestions followed from predictive coding models of learning and inference (Friston, 2005; Rao and Ballard, 1999) and their specific application to schizophrenia (Stephan et al, 2006). Predictive coding assumes a hierarchical neural architecture where each level provides predictions about the state of the level below and evaluates the discrepancy with the actual inputs from the lower level, that is, prediction error (PE) (Friston, 2005; Rao and Ballard, 1999). Predictions and PE are conveyed via synaptic connections instantiating a Bayesian message passing scheme. Simply speaking, both perceptual inference and learning rely on minimization of $\mathrm{PE}$ throughout the hierarchy. Neuronal states and connection strengths are adjusted during inference and learning, respectively, to minimize PE at all levels of the hierarchy.

It has repeatedly been proposed that aberrant $\mathrm{PE}$ processing might be involved in the formation of psychosis, originally in the context of associative learning theory (Gray et al, 1991; Hemsley, 1993) and more recently in terms of Bayesian concepts (Fletcher and Frith, 2009; Murray et al, 2008; Stephan et al, 2006). Broadly speaking, these theories constitute two non-exclusive classes that stress either (i) inappropriate timing of PE signals (King et al, 1984; Shaner, 1999) or (ii) inadequate magnitude or precision (inverse variance) of $\mathrm{PE}$ estimates during hierarchical Bayesian inference (Corlett et al, 2009; Friston, 2005; Stephan et al, 2006). These general concepts have been used to explain a range of empirical findings about schizophrenia, such as interpreting fMRI data in terms of a disruption of PE processing in the PFC of schizophrenia patients with delusions (Corlett et al, 2007). Critically, the extent of this PE disruption was related to an individual's propensity to delusion formation. Another example is that in the early stage of psychosis, schizophrenia patients often describe how irrelevant stimuli capture their attention (Chapman, 1966). This has been framed in terms of 'aberrant salience', a phenomenological concept originally developed without explicit reference to PEs (Kapur, 2003). More recent proposals (Roiser et al, 2009) have speculated about a link of aberrant salience to contextually irrelevant or 'chaotic' PEs (King et al, 1984), which may underlie formation of inappropriate statistical associations, leading to maladaptive inference about the causes of sensory inputs. In summary, abnormal PEs may represent a general mechanism for the emergence of psychotic symptoms in schizophrenia and understanding these symptoms requires formal models that link cognitive impairments to psychopathology.

A commonly used physiological paradigm to estimate $\mathrm{PE}$ signaling is the mismatch negativity (MMN) event-related potential (ERP) (Garrido et al, 2009b; Todd and Robinson, 2010), which is most relevant to psychotic symptoms in naturally occurring and drug-induced psychosis (Corlett et al, 2011; Kantrowitz and Javitt, 2010). MMN is an electrophysiological event-related response to unexpected sensory (typically auditory) stimuli, the so-called 'deviants', that differ in one physical stimulus dimension from a sequence of preceding identical 'standards' (Näätänen $e t$ al, 1978). Indeed, deficits in MMN amplitude have been repeatedly reported in schizophrenic patients (Umbricht and Krljes, 2005), and a comparable deficit was found in healthy volunteers after exposure to ketamine (Heekeren et al, 2008; Umbricht et al, 2000). Furthermore, the MMN amplitude assessed under placebo correlated positively with the ketamine-induced positive-like symptoms as indexed by the global score of the Altered State of Consciousness rating scale (5D-ASC) and the BPRS psychosis score in healthy subjects (Umbricht $e t a l, 2002$ ). This correlation was significant over frontal electrodes, suggesting that the integrity of PFC is crucial for PE processing in MMN. Another study using a different modality (fMRI) and different paradigm (an associative learning task) suggested that this role of the PFC for PE processing may generalize, reporting that ketamine disrupted PE-dependent activity in the right PFC of healthy volunteers (Corlett et al, 2006).

In this study, we investigated how $\mathrm{PE}$ processing is altered under NMDAR antagonist and/or 5- $\mathrm{HT}_{2 \mathrm{~A}} \mathrm{R}$ agonist manipulation, and whether ketamine- and psilocybin-induced symptoms can be predicted by the MMN. Instead of a classical oddball paradigm, we use a 'roving' MMN paradigm and examine the formation and strengthening of memory traces (by quantifying the amplitude of the MMN as a function of the number of preceding standards) (Haenschel et al, 2005; Imada et al, 1993). A notable advantage of the roving paradigm is that physical stimulus differences between standards and deviants are varied independently of the number of standard repetitions in the roving paradigm. This ensures that the ensuing MMN memory trace effect is entirely due to learning and cannot result from differential states of frequency-specific auditory neurons in the temporal cortex.

\section{MATERIALS AND METHODS}

This study was approved by the Ethics Committee of the University Hospital of Psychiatry, Zurich, Switzerland. After receiving a written and oral description of the aim of this study, all participants gave written informed consent statements before inclusion. The use of psychoactive drugs was approved by the Swiss Federal Health Office (BAG), Department of Pharmacology and Narcotics (DPN), Bern, Switzerland.

\section{Subjects}

Healthy subjects were recruited at the local university and technical college through advertisement and were then divided into two groups, either receiving $S$-ketamine or psilocybin (ketamine group: $N=19$ (male: 12), mean age $=26 \pm 5.09$ years; psilocybin group: $N=20$ (male: 12 ), mean age $=23 \pm 2.27$ years).

Before the inclusion, subjects' physical health was confirmed by medical history, clinical examination, electrocardiography, and blood analysis. To ascertain the subjects' mental status, all subjects were screened by the DIA-X diagnostic expert system (Wittchen and Pfister, 1997), a semi-structured psychiatric interview, and the Hopkins Symptom Checklist (SCL-90-R) (Derogatis, 1994). Furthermore, subjects replied to the Mini-International Neuropsychiatric Interview (MINI.), a short, structured psychiatric interview (Sheehan et al, 1998). We verified the absence of a history of drug dependence or present drug abuse by urine drug screening and a questionnaire of drug consumption. 


\section{Drug Administration}

In both groups, subjects underwent two sessions (placebo/ active drug) in a counterbalanced order. Both subjects and principal investigator were blind to drug order. Subjects stayed monitored and under constant supervision until all drug effects had worn off, and were then released into the custody of a partner or immediate relative.

For the $S$-ketamine/placebo infusion, an in-dwelling catheter was placed in the antecubital vein of the nondominant arm. The infusion scheme can be summarized as BET (Krüger-Thiemer, 1968). Once the subject was ready, a bolus injection over $5 \mathrm{~min}$ with $10 \mathrm{mg}$ of ketamine was given (B). Following $1 \mathrm{~min}$ break, a continuous infusion with $0.006 \mathrm{mg} / \mathrm{kg}$ per min was started (E). To keep $S$-ketamine levels fairly constant and prevent accumulation in the brain, the dose was reduced by titrating $(\mathrm{T})$. In the placebo session, the same procedure was followed. Instead of $S$-ketamine, an infusion of physiological sodium chloride solution and $5 \%$ glucose was given. Psilocybin was given per os at a dose of $115 \mu \mathrm{g} / \mathrm{kg}$. The specific doses were chosen based on previous studies (Kometer et al, 2011; Vollenweider and Kometer, 2010).

\section{Psychometric Assessment of $S$-Ketamine- and Psilocybin-Induced States (ASC-R)}

A revised form of the Altered State of Consciousness (ASC-R) questionnaire, a visual analogue and self-rating scale, was used to assess the subjective effects of ketamine and psilocybin (Dittrich, 1975, 1998). This revised scale (ASC-R) (Studerus et al, 2010) comprises three primary dimensions and their respective subscales: (i) 'oceanic boundlessness', referring to positively experienced egodissolution associated with changes in the sense of time and emotions, ranging from heightened mood to sublime happiness and feelings of unity with the environment, subsuming the subscales disembodiment, blissful state, spiritual experience, and experience of unity and insightfulness; (ii) 'anxious ego-disintegration', comprising the subscales anxiety and impaired control and cognition, including items for disordered thought and loss of control over body and thought; and (iii) visionary restructuralization, including the subscales elementary and complex imagery, audio-visual synesthesias, changed meaning of percepts, and auditory alterations.

\section{Auditory Test Paradigm (MMN)}

Electroencephalographic (EEG) activity was measured during an auditory 'roving' oddball paradigm (Baldeweg et al, 2004; Boly et al, 2011; Garrido et al, 2008; Haenschel et al, 2005), originally developed by Cowan et al (1993). Acoustic stimuli were generated by the E-prime software (Schneider et al, 2002), and applied binaurally through headphones (TDH-39-P; Maico, Minneapolis, MN).

Stimuli comprised a structured train of pure sinusoidal tones, with a roving changing tone (Figure 1). Within each stimulus train, all tones were of one frequency and were followed by a train of a different frequency. The first tone of a train represents the deviant (red), which becomes the standard tone after a few repetitions (blue). Hence, the deviants and standard tones have exactly the same physical properties within one stimulus train, differing only in the number of times they have been presented in the recent past (in a previous stimulus train). The number of times the same tone was presented within one stimulus train varied pseudo-randomly between 1 and $11(t=1-11)$. The probability that the same tone was presented in one stimulus train (i) was $2.5 \%$ for trains with 1 or 2 identical tones, (ii) $3.75 \%$ for trains with 3 or 4 identical tones, and (iii) $12.5 \%$ for trains with 5-11 identical tones. In other words, $5 \%$ of all stimulus trains consisted of 1-2 identical tones, $7.5 \%$ of all stimulus trains consisted of 3-4 identical stimuli, and $87.5 \%$ of all stimulus trains consisted of $5-11$ identical stimuli. The frequency of the tones varied from 500 to $800 \mathrm{~Hz}$ in random steps with integer multiples of $50 \mathrm{~Hz}$, tone duration was set at $70 \mathrm{~ms}$, and the inter-stimulus interval was $500 \mathrm{~ms}$.

In parallel, subjects performed a distracting visual task and were instructed to ignore the sounds. It has been argued

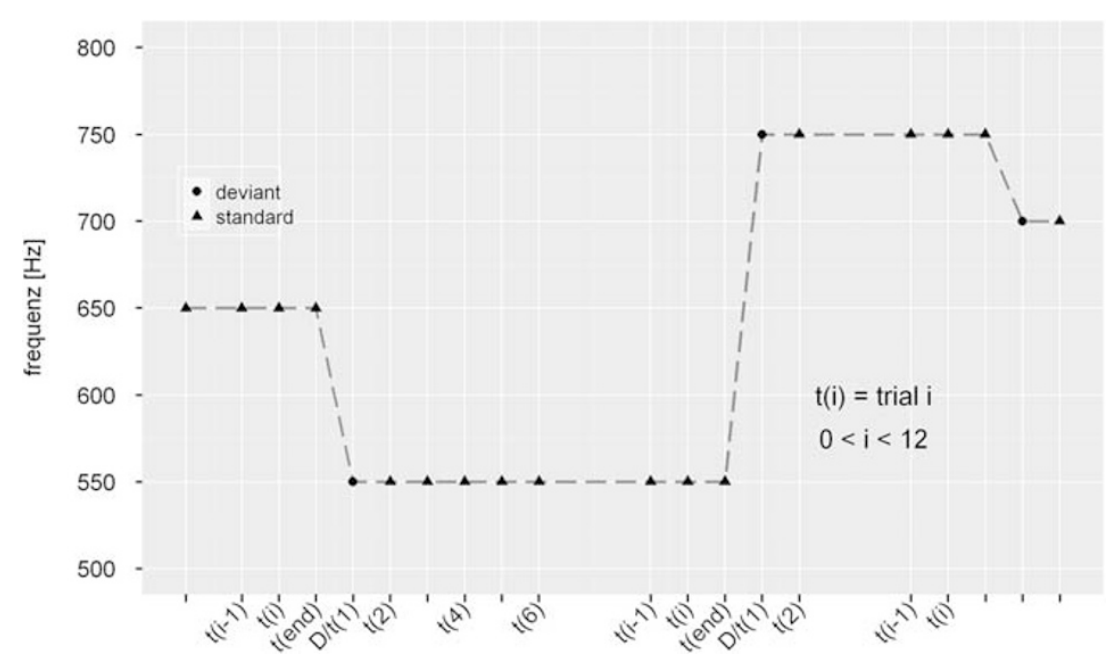

Figure I Stimulus design is characterized by a sporadically changing standard stimulus. The first presentation of a novel tone is a deviant (D/t( $I)$; circle) that becomes a standard (triangle), through repetition $(t(6))$. Standard repetition varied between I and II $(i=|-| \mathrm{I})$. 
that the best condition to observe an MMN is when the subject's attention is directed away from the stimulus (Näätänen, 2000). The task consisted of button-pressing whenever a fixation cross-changed its luminance, which occurred pseudo-randomly every $2-5 \mathrm{~s}$ (not coinciding with auditory changes). The testing session lasted approximately $15 \mathrm{~min}$. The purpose of the additional distracting task was to ensure that the participant's attention was not focused on the tones.

\section{EEG/ERP Recording}

EEG recordings were made from 64 scalp electrodes using the ActiveTwo system (Biosemi, Amsterdam, The Netherlands). The horizontal electroocculogram (EOG) was recorded from electrodes attached on the outer canthus of each eye. Similarly, vertical EOG was recorded from electrodes attached infraorbitally and supraorbitally to the left eye. Data were recorded at a sampling rate of $512 \mathrm{~Hz}$. All electrodes were active silver/silver chloride electrodes and were referenced during recording to a common-mode signal electrode between $\mathrm{POz}$ and $\mathrm{PO} 3$ (see http://www. biosemi.com/faq/cms\&drl.htm for more details on this setup); the offset of all electrodes was below $25 \mathrm{mV}$, indicating good recording quality.

For ERP analysis, independent component analysis (ICA) was used to remove artifacts due to eye movements and blinks. Following ICA, epochs with a 200-ms prestimulus baseline and a 500-ms poststimulus interval were constructed. Epochs with amplitudes that exceeded $\pm 100 \mu \mathrm{V}$ at any electrode were excluded from further averaging. Following artifact rejection, epochs were averaged offline for each subject and were digitally filtered with a band-pass filter $(1-30 \mathrm{~Hz})$. MMN was measured as the peak negativity within the 100-200 ms window latency of difference waveform (deviant-standard waveforms). Here, we followed previous studies of the roving paradigm (eg, Garrido et al, 2008) in focusing only on deviants preceded by at least 6 standards. Waveforms were mathematically referenced to an average-mastoid reference before peak detection, analogously analyzed as previous studies (Umbricht et al, $2000,2002,2003)$. Based on previous studies, a set of frontocentral (Fz, F3, and F4) and -temporal electrodes (TP7 and TP8) were preselected (Alho et al, 1996; Sato et al, 2000; Tiitinen et al, 1993; Waberski et al, 2001).

\section{Statistical Analysis}

All statistical analysis was conducted using Statistica 7.1 for Windows (Statsoft, Tulsa, OK, USA). Results of the distracting visual task were evaluated according to signal detection theory (Macmillan and Creelman, 1991) to determine sensitivity indexes $\left(d^{\prime}\right)$ under placebo and following drug administration, which were entered into a repeated-measures analysis of variance (repeated-measures ANOVA) with between-subjects factor group (ketamine group $v s$ psilocybin group). Furthermore, averaged means of MMN difference wave amplitude and latency were subjected to a repeated-measures ANOVA using the within-subjects factors, electrode (frontal vs temporal), treatment (placebo $v s$ drug), and standard repetition $(t=6,8$, and 10), and the between-subjects factor group (ketamine group vs psilocybin group). Where the ANOVA null hypothesis of equal means was rejected, we used posthoc tests (Fisher's least significant difference (LSD) test). In addition, correlations were conducted to examine the effect of the distracting visual task ( $d^{\prime}$ values) on the MMN difference wave amplitudes. Another repeated-measures ANOVA was carried out to study ketamine- and psilocybininduced symptoms.

For further analysis, the relative increase of the MMN difference waveform from $t(6)$ to $t(8)$ and further from $t(8)$ to $t(10)$ was computed and the average of these values expressed as 'MMN slope'. To assess the relationship between MMN slope under 'baseline' (drug-free) conditions and drug-induced psychopathology, we correlated (over subjects) the MMN slope under placebo with each symptom rating in the ASC-R questionnaire and Bonferroni corrected the significance level for multiple testing.

\section{RESULTS}

\section{Distracting Visual Vigilance Test}

Repeated-measures ANOVA revealed that both drugs produced significantly prolonged reaction times $(\mathrm{F}(1,37)=$ 31.4, $\left.P<0.0000, \eta^{2}=0.46\right)$ as well as reduced sensitivity indices $\left(d^{\prime}\right)\left(\mathrm{F}(1,37)=20.8, P<0.00001, \eta^{2}=0.36\right)($ Table 1$)$. Drug effects did not differ from each other, as indicated by the lack of interaction with the between-subjects factor group.

\section{MMN Memory Trace Effect (PE Processing)}

A significant main effect for standard repetition revealed the systematic increase in MMN amplitude with increasing number of standard tones (memory trace effect) $\left(\mathrm{F}(2,74)=3.58, P<0.05, \eta^{2}=0.1\right)$. In general, both overall MMN amplitude and MMN trace effect were more pronounced over frontal than temporal electrodes $(P<0.000001)$, as indicated by a significant main effect for electrode $\left(\mathrm{F}(1,37)=145, \quad P<0.000001, \quad \eta^{2}=0.8\right)$ and a significant electrode $\times$ standard repetition interaction $\left(\mathrm{F}(2,74)=5.1, P<0.01, \eta^{2}=0.12\right)$. Furthermore, a main effect of treatment was found $(\mathrm{F}(1,37)=8.35, P<0.01$, $\left.\eta^{2}=0.18\right)$ reflecting an overall attenuation of the MMN amplitude, irrespective of standard repetition, following drug administration. Furthermore, a treatment $\times$ electrode interaction $\left(\mathrm{F}(1,37)=5, P<0.05, \eta^{2}=0.12\right)$ revealed that this treatment effect occurred only over frontal electrodes $(P<0.00000)$. However, the electrode $\times$ treatment $\times$ group

Table I Behavioral Results of the Distracting Visual Vigilance Task

\begin{tabular}{|c|c|c|c|c|c|c|}
\hline & \multicolumn{3}{|c|}{ Ketamine group } & \multicolumn{3}{|c|}{ Psilocybin group } \\
\hline & Placebo & Ketamine & $P$-value & Placebo & Psilocybin & $P$-value \\
\hline $\mathrm{RT}(\mathrm{ms})$ & $331 \pm 4.3$ & $350 \pm 5.2$ & 0.001 & $340 \pm 4.6$ & $360 \pm 5.1$ & 0.001 \\
\hline$d^{\prime}$ & $1.9 \pm 0.27$ & $1.1 \pm 0.22$ & 0.01 & $1.6 \pm 0.28$ & $0.8 \pm 0.12$ & 0.01 \\
\hline
\end{tabular}

Mean reaction times $(\mathrm{RT})$ and sensitivity indices $\left(d^{\prime}\right) \pm$ SE. Both measures were significantly worsened by both drugs, but did not differ significantly between drugs. 
interaction revealed that this treatment effect depended on the location of the electrodes and on the specific drug used $\left(\mathrm{F}(1,37)=10.5, P<0.01, \eta^{2}=0.22\right)$. Although psilocybin did not affect the MMN over frontal or temporal electrodes, ketamine did disrupt the MMN, but only at frontal electrodes $(P<0.00001)$ (Figure 2). A subsequent ANOVA of the ketamine group revealed that ketamine disrupted the MMN memory trace effect over frontal electrodes, indicated by a triple electrode $\times$ treatment $\times$ standard repetition interaction $\left(\mathrm{F}(2,36)=4.7, P<0.05, \eta^{2}=0.21\right)$. Post-hoc analysis showed that for the frontal electrodes Fz, F3, and F4 shown in Figure 2, the treatment $\times$ standard repetition interaction was also significant $(P$ at $t(6)<0.0001 ; P$ at $t(8)<0.0001$; $P$ at $t(10)<0.000001)$, indicating that the difference in the effect of ketamine $v s$ placebo on the MMN trace effect became more pronounced with longer trace length. The corresponding MMN difference waves for the ketamine group were plotted in Figure 3. Finally, as shown by a significant treatment $\times$ group interaction (Figure 3), ketamine but not psilocybin caused a latency shift of the MMN, irrespective of standard repetition $(\mathrm{F}(1,37)=5.29, P<0.05$, $\left.\eta^{2}=0.13\right)$.

Notably, no significant correlations between the behavioral results of the distracting visual vigilance task ( $d^{\prime}$ values) and the MMN slope in either group were found, suggesting a functional dissociation.

\section{$S$-Ketamine- and Psilocybin-Induced Symptoms (ASC-R Questionnaire)}

At the dose tested, both ketamine and psilocybin produced similar alterations on the global ASC score that were characterized by derealization and depersonalization phenomena, affective changes, cognitive impairments, and perceptual alterations, reflected by a main effect for treatment $\left(\mathrm{F}(1,37)=69.5, \quad P<0.000001, \quad \eta^{2}=0.65\right)$.
Subsequent ANOVA with treatment and subscale as repeated measures and group as between-subject factor revealed that both drugs significantly increased all subscale scores (all P's $<0.01$ ), with the exception of the anxiety score $\left(\mathrm{F}(11,407)=4.6, P<0.00001, \eta^{2}=0.11\right)$. In addition, psilocybin-induced auditory alterations were not

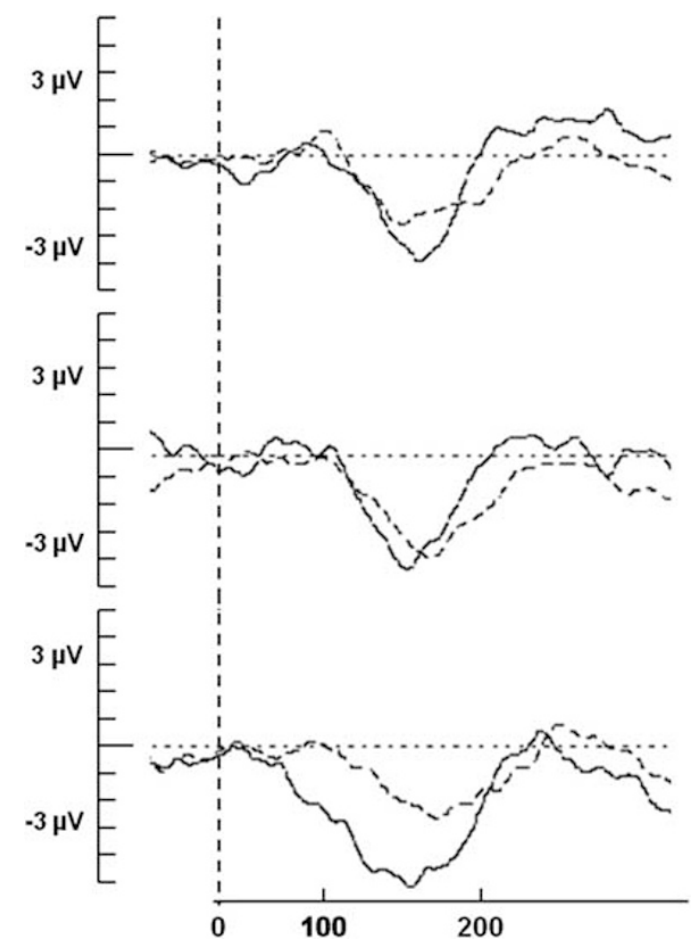

Figure 3 Corresponding mismatch negativity (MMN) difference waveforms following placebo (solid line) and ketamine administration (dashed line) at frontal electrodes (Fz, F3, and F4) for $t(6)$ (above), $t(8)$ (middle), and $t(10)$ (below).

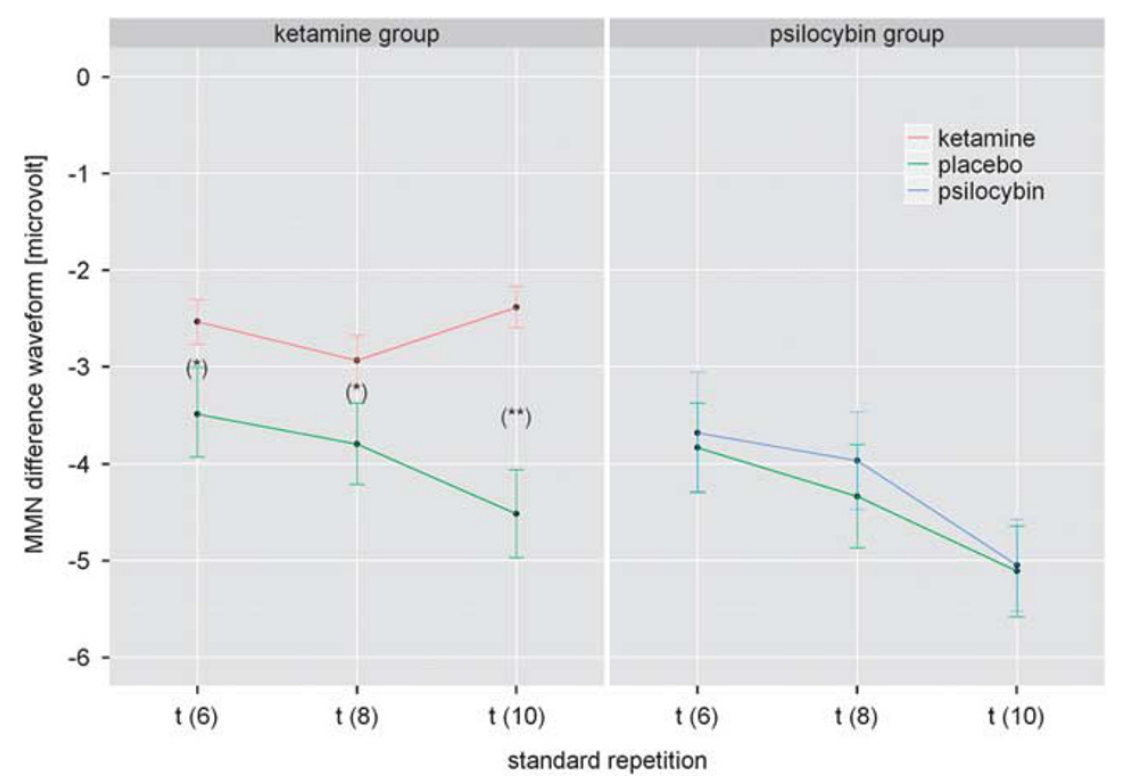

Figure 2 Mean amplitudes \pm SE of mismatch negativity (MMN) difference waveforms across standard repetitions $(t=6-10)$ at frontal electrodes (Fz, F3, and F4) following placebo (green) or drug administration (red: ketamine; blue: psilocybin), respectively. Note: Significant differences between treatment conditions at $* P<0.000$ I and at $* * P<0.000001$. 


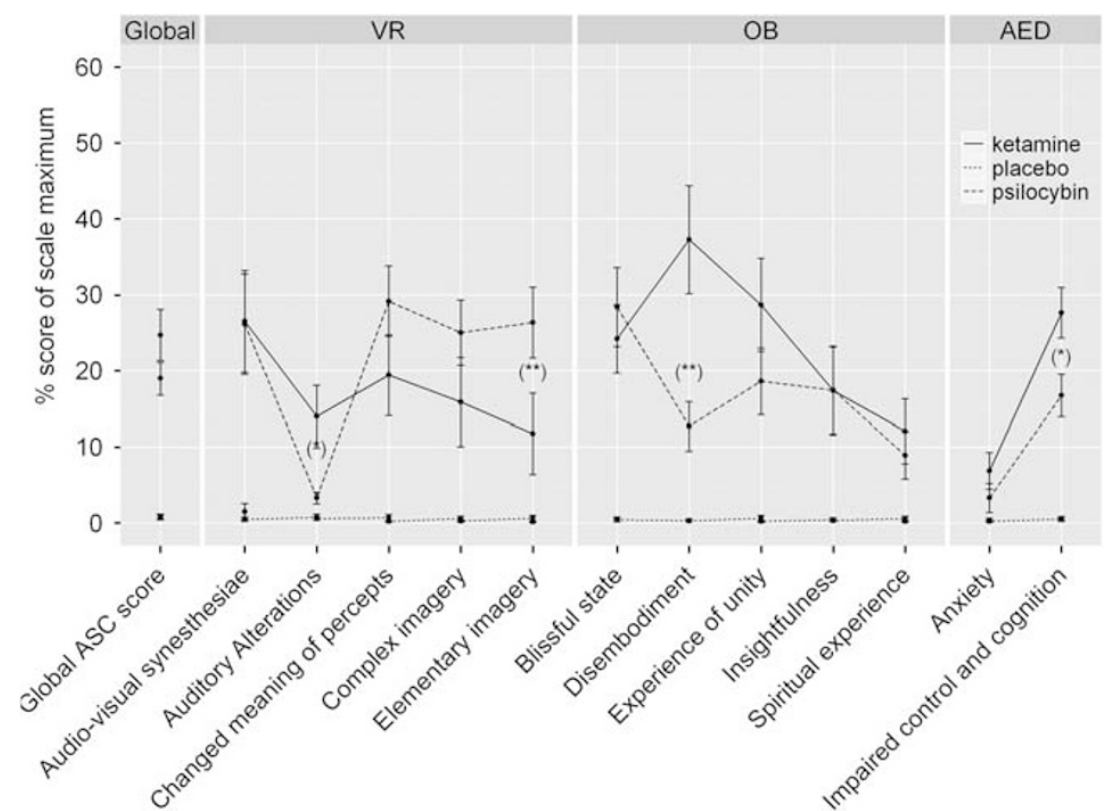

Figure 4 Means \pm SE of ketamine- (solid line) and psilocybin-induced (dashed line) symptoms relative to placebo (dotted line) measured with the revised form of the Altered State of Consciousness (ASC-R). Note: Significant differences (least significant difference (LSD) test) between drugs at $* P<0.05$ and at ** $P<0.0$ I. Symptoms scores were expressed as the percent of scale maximum. The abbreviation VR comprised 'visionary restructuralization', OB means 'oceanic boundlessness', and AED stands for 'anxious ego-disintegration'. Both ketamine and psilocybin increased the global ASC score relative to placebo, but did not differ from each other. Ketamine produced higher score than psilocybin for auditory alterations, disembodiment, and impaired control and cognition. Otherwise, psilocybin produced higher score for elementary imagery than ketamine. No correlation between drug-induced cognitive impairments (impaired control and cognition) and drug-induced perceptual alterations (auditory and visual) was found.

significantly higher than that under placebo. Moreover, post-hoc analysis showed that ketamine produced significantly higher scores than psilocybin for auditory alterations $(P<0.05)$, for depersonalization as indexed by disembodiment $(P<0.000001)$ and for cognitive impairments as indexed by impaired control and cognition $(P<0.05)$ (Figure 4). Otherwise, psilocybin produced more severe elementary imagery $(P<0.01)$, including visual illusions and (pseudo-) hallucinations than ketamine. Notably, no correlation was found between drug-induced cognitive impairments (impaired control and cognition) and druginduced perceptual alterations (auditory and visual). This speaks against the possibility that drug-induced perceptual aberrations induced cognitive impairments.

\section{Linking MMN Slope and Drug-Induced Symptoms}

We assessed the relation between MMN slope under 'baseline' (drug-free) conditions at frontal electrodes and drug-induced psychopathology by correlating the MMN slope under placebo with each symptom rating from the ASC-R questionnaire (under Bonferroni correction for multiple testing). We found a significant correlation between the MMN slope under placebo and ratings pertaining to impaired control and cognition (which includes items for disordered thought and loss of control over body and thought). Critically, this correlation was significant under ketamine $(r=-0.67 ; P<0.05$ Bonferroni corrected), but not under psilocybin $(r=-0.11 ; P=\mathrm{NS})$ (Figure 5). Those subjects who showed the greatest MMN slope under placebo experienced the lowest ketamineinduced cognitive impairments. No other correlations between the MMN slope under placebo and any other drug-induced symptom was found.

\section{DISCUSSION}

Our study provides two major results: Firstly, prefrontal PE processing during the roving MMN paradigm is disrupted by the NMDAR antagonist $S$-ketamine, but not by the $5-\mathrm{HT}_{2 \mathrm{~A}} \mathrm{R}$ agonist psilocybin. Secondly, the processing of PE under placebo predicts the severity of ketamine-induced cognitive impairments. In the following, we discuss these findings within a predictive coding framework of MMN (Baldeweg, 2006; Friston, 2005; Garrido et al, 2009a,b), relating them to putative neurobiological mechanisms for the emergence of cognitive impairments.

According to classical theories (Näätänen, 1992), the MMN potential originates from a network comprising the superior temporal gyrus (STG) and the inferior frontal gyrus (IFG) (Garrido et al, 2008; Opitz et al, 2002; Rinne et al, 2000), which detects a disruption within a regularity of preceding standards based on a comparison of the actual sensory input with the already established memory trace (Doeller et al, 2003; Giard et al, 1990). In this study, we have performed an analysis in sensor space; since EEG sensor signals result from a mixture of neuronal sources, one should be cautious about relating our findings to neural sources in specific locations. In other words, an MMN effect observed at frontal electrodes must not necessarily correspond to a frontal source, and the following discussion should be read with this caveat in mind. 


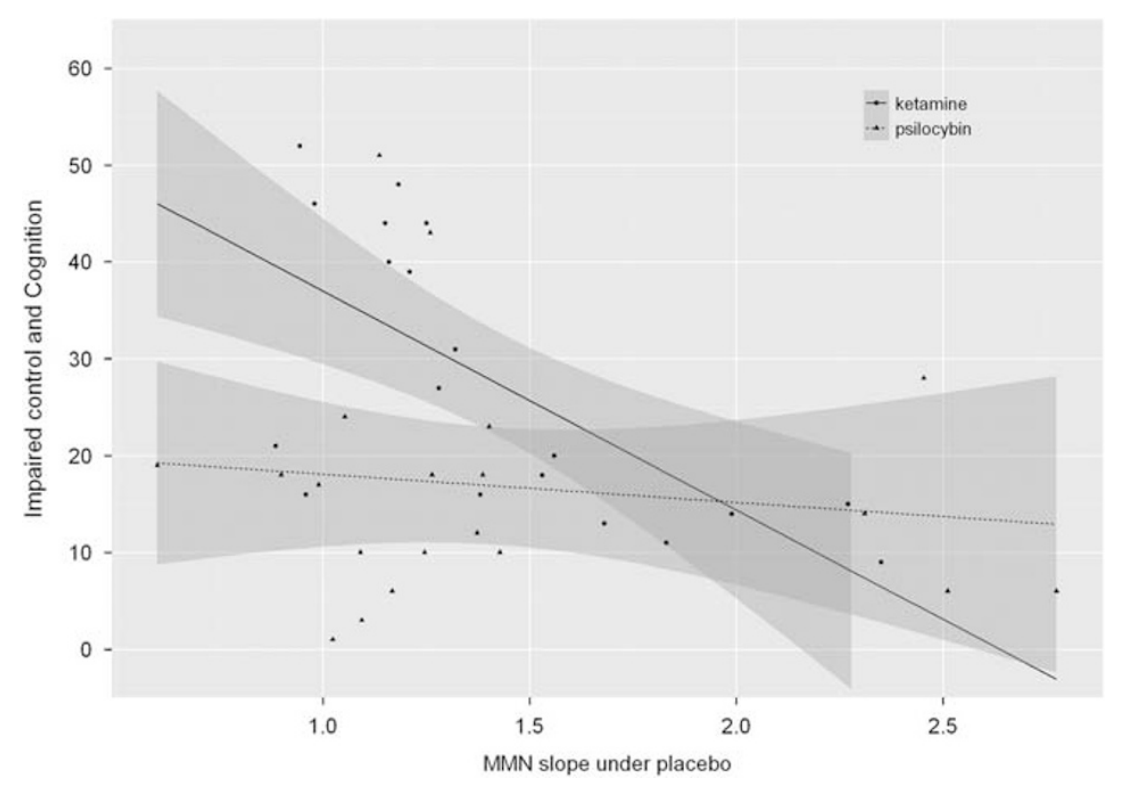

Figure 5 Relationship between the mismatch negativity (MMN) slope under placebo over frontal electrodes and the subscale impaired control and cognition, which includes disordered thought and loss of control over body and thought, induced by ketamine (solid line) and psilocybin (dotted line). Impaired control and cognition scores are expressed as the percentage of the maximum points possible. Notably, the MMN slope under placebo was predictive of ketamine-induced cognitive impairments $(r=-0.67 ; P<0.05)$, but not of psilocybin-induced cognitive impairments $(r=-0.11$; $P=N S)$.

Consistent with other reports using a 'roving' oddball design (Baldeweg et al, 2004; Haenschel et al, 2005), an MMN memory trace effect was found in this study over the frontal cortex; however, no comparable effect was detected over temporal cortical regions. The absence of a temporal MMN memory trace effect suggests that the adaptation hypothesis (Jääskeläinen et al, 2004), which posits that the MMN results from local neuronal adaptation in the auditory cortex, cannot easily explain the MMN memory trace effect found in this study. Instead, the frontal MMN memory trace effect may be more plausibly understood as a marker of PE caused by the deviation from a learned regularity. This interpretation would be in line with the model adjustment hypothesis (Näätänen and Winkler, 1999; Winkler et al, 1996). However, another roving MMN study in healthy volunteers (which focused on MMN amplitudes in reference to the 6th standard and did not quantify memory trace effects) used dynamic causal modeling and Bayesian model selection to suggest that the model explaining MMN amplitude best was a combination of the adaptation and model adjustment hypotheses. This model accommodates intra-areal adaptation within the STG combined with plasticity of inter-areal connections between temporal and frontal regions (Garrido et al, 2008). The authors explicitly interpreted their findings within the predictive coding framework, emphasizing that this theory includes both adaptation and model adjustment in the sense that 'model adjustment' corresponds to the adjustment of a generative model of future stimuli and 'adaptation' corresponds to local changes in post-synaptic gain (Garrido et al, 2008). Thus, from this perspective, the MMN would arise from a failure to predict bottom-up input and suppress the resulting PE (Garrido et al, 2008, 2009b).

As reported previously (Heekeren et al, 2008; Umbricht et al, 2000), the temporal MMN (TP7/TP8) was not significantly altered by $S$-ketamine in this study, although this lack of effect may have resulted from the chosen nearby mastoid (TP9/10) reference, which might mask a drug effect on this component. However, similar to this study, no disruption of the temporal MMN was observed in schizophrenic patients $v s$ healthy controls in two recent studies using the nose as reference and applying a similar 'roving' paradigm as used in this study (Baldeweg et al, 2004; Sato et al, 2003). In contrast to $S$-ketamine's lack of effect on temporal MMN, our study found that $S$-ketamine did disrupt the frontal MMN, which is consistent with previous human studies using different MMN paradigms (Heekeren et al, 2008; Umbricht et al, 2000). Here we extend these previous findings by showing that $S$-ketamine not only reduced the MMN amplitude, but also disrupted the frontally generated MMN memory trace effect compared with placebo. A similar reduction of the frontal memory trace effect was observed in schizophrenic patients (Baldeweg et al, 2004). This finding, together with the established importance of the NMDAR for synaptic plasticity during perceptual learning (Kandel, 2001; Morris et al, 1986), fits nicely to the role that neurobiologically specific predictive coding theories of MMN have assigned to NMDARdependent plasticity. In these theories (Friston, 2005; Stephan et al, 2006), NMDAR-dependent short-term plasticity is critical for adjusting the strength of glutamatergic synapses connecting hierarchically related cortical areas during perceptual learning. Computationally, this synaptic adjustment is driven by the magnitude of trial-wise PEs in lower-level structures, leading to adjustment of predictions generated by higher-level structures. From this perspective, our finding of a reduced frontal MMN memory trace may reflect the drug-induced perturbation of NMDAR-dependent plasticity of temporo-frontal (forward) connections that serves to adjust PE message passing between the STG and the IFG during MMN generation (Garrido et al, 2008; Opitz et al, 2002). In other words, the 
disruption of frontal MMN by $S$-ketamine may result from a deficient adjustment of prefrontally generated predictions about temporal inputs due to insufficient PE-dependent plasticity of forward connections. We will examine the plausibility of this potential mechanism in future modeling studies. However, the present data cannot fully exclude that increased AMPA receptor conductance following NMDAR blockade (Shi and Zhang, 2003) or downstream effects on the dopaminergic system (Kapur and Seeman, 2001; Vollenweider et al, 2000) may also have contributed to the present findings. Indeed, in the context of predictive coding accounts of psychosis, it was suggested recently that both NMDAR (for backward connections) and AMPAR (for forward connections) contribute to conveying PE information (Corlett et al, 2009). In contrast, the empirical data from studies examining the MMN under dopaminergic manipulations indicate that dopamine does not modulate the MMN (Korostenskaja et al, 2008; Leung et al, 2007; Leung et al, 2010).

Some symptoms frequently observed in schizophrenia are not typically interpreted within a Bayesian framework and are usually examined from different perspectives. For example, this include deficits in early information processing as indexed by measures as prepulse inhibition of startle reflex or the P50 auditory-evoked potential suppression (Braff et al, 2001; Turetsky et al, 2007). In this context, it has been recently suggested that in schizophrenic patients, deficits in early sensory processing may explain the observed reduction in MMN (Leitman et al, 2010). If one accepts $S$-ketamine as a valid model of schizophrenic symptoms, then this may be seen as a challenge for our findings. However, given that physical differences between deviant and standard tones were independent of the number of standard tones in the roving paradigm and that the difference in the effect of ketamine vs placebo on the MMN trace effect became more pronounced with longer trace length, it seems unlikely that a pure deficit in early sensory processing could account for the ketamine-induced disruption of the MMN memory trace effect. This interpretation is further supported by a lack of correlation between ketamine-induced auditory alterations and the generation of the MMN memory trace effect.

Contrary to $S$-ketamine, the $5-\mathrm{HT}_{2 \mathrm{~A}} \mathrm{R}$ agonist psilocybin neither reduced the MMN amplitude nor the MMN memory trace effect, which is consistent with a previously reported lack of psilocybin on MMN measured by a classical paradigm (Umbricht et al, 2003). Similarly, a recent MMN study with $S$-ketamine and the $5-\mathrm{HT}_{2 \mathrm{~A}} \mathrm{R}$ agonist DMT showed that the frontal MMN was only affected by S-ketamine and not by DMT (Heekeren et al, 2008). Although $5-\mathrm{HT}_{2 \mathrm{~A}} \mathrm{R}$ seems to be involved in different forms of working memory (Williams et al, 2002) and $5-\mathrm{HT}_{2 \mathrm{~A}} \mathrm{R}$ agonists such as LSD or DOM enhanced associative learning as measured by eyeblink conditioning in rabbits (Harvey, 2003), the implicit perceptual learning as indexed by the frontal memory trace effect appeared not to be sensitive to manipulations of the $5-\mathrm{HT}_{2 \mathrm{~A}} \mathrm{R}$ system (or to the dose of psilocybin used in this study). Although it has been proposed that neuromodulators like serotonin may influence the processing of PE (Corlett et al, 2009) and may be one of the key modulators interacting with NMDARs to produce aberrant synaptic plasticity in schizophrenia (Stephan et al, 2009), this does not necessarily contradict our findings since such a putative role of serotonin could be expressed in a regionally specific manner (ie, in other circuits than the auditory system), and may thus not be a critical factor in $\mathrm{PE}$ processing during the MMN paradigm (cf. Garrido et al, 2009b).

Furthermore, we also explored whether ketamine- or psilocybin-induced symptoms could be predicted by the MMN slope (in the absence of any drug) (ie, under placebo); in other words, we used two pharmacological models of psychosis to relate individual 'baseline' physiology to his/her predisposition to develop psychotic symptoms under pharmacological challenge. This approach is inspired by the general notion that the development of psychiatric disease may result from the interplay between the (genetically determined) susceptibility of an individual and the expose to environmental stressors (Brown, 2011), and follows previous studies, which examined whether physiological markers can predict the individual degree of psychotic symptoms in drug-induced psychosis and schizophrenia (Corlett et al, 2006; Honey et al, 2008; Krystal et al, 2003; Umbricht et al, 2002). Here, we found that the MMN slope under placebo showed a negative correlation with the subsequent ketamine-induced extent of cognitive impairments across subjects; in contrast, no comparable relationship was found for psilocybin. Although this result is in accordance with Umbricht's MMN study (Umbricht et al, 2002), it may not appear to be fully consistent with the results by Corlett et al (2006), who found that frontal PE signals under placebo, measured during an associative learning task with fMRI, exhibited a positive correlation with the severity of positive symptoms (delusions, perceptual aberrations) under ketamine. However, the results from the two studies are not directly comparable owing to a number of major methodological differences, including different symptom rating scales, different ketamine dosage and application regimens, different measurement techniques (fMRI vs EEG), and, perhaps most importantly, fundamentally different cognitive paradigms. Still, one may wonder why individual MMN slope under placebo should predict ketamine-induced cognitive impairments (rather than other symptoms). This can be understood by considering that ketamine disrupts (short- and long-term) NMDARmediated synaptic plasticity, which is a crucial mechanism for PE-dependent learning (for a review, see Stephan et al (2006)). This is relevant for understanding cognitive impairments or 'negative symptoms', such as thought disorder, in schizophrenia because 'many, if not all, of them can also be understood as a consequence of aberrant modulation of synaptic plasticity' (Stephan et al, 2009). In brief, if one is endowed with inadequate PE-dependent learning (due to NMDAR-related deficiencies), one may be impaired in learning about the statistical structure of the (social and physical) environment and adapting one's beliefs and behavior accordingly. This could explain a wide range of symptoms, from social withdrawal and apathy to thought disorders and aberrant perceptual inference (see Stephan et al (2009) for details). On the other hand, the magnitude of the MMN slope can be seen as expressing the individual capacity for PE-dependent learning, that is, how trial-wise 'surprise' about a deviant induces NMDAR-dependent shortterm plasticity to update predictions about the next trial. 
In other words, MMN slope may serve as an index of individual capacity of utilizing PEs for adaptive cognition through NMDAR-dependent plasticity. From this perspective, one would predict that the higher this individual capacity in the drug-free state (ie, the higher the MMN slope under placebo), the less pronounced the effects of ketamine on PE-dependent learning and subsequent aberrations in adaptive cognition. This is what we found.

To conclude, these results suggest that the frontal MMN memory trace effect may provide a useful approach to study NMDAR-dependent PE processing during the MMN as a form of implicit perceptual learning. Unraveling the role of NMDAR function in predictive coding may provide valuable insights into pathophysiological mechanisms of schizophrenia in general and the emergence of cognitive impairments in psychosis in particular. This may particularly benefit from a computational modeling approach, which uses physiologically interpretable model parameters for clinical predictions (Stephan et al, 2006). In relation to this, recent studies demonstrated that a reduction of MMN can predict the transition of 'ultra-high risk' to first-episode psychosis (Bodatsch et al, 2010; Shin et al, 2009). Finally, the assessment of the MMN memory trace effect may also provide a promising tool to assess the efficacy of novel pharmacological treatment, in particular for treatment of cognitive impairments.

\section{ACKNOWLEDGEMENTS}

This study was supported by the Swiss Neuromatrix Foundation (AS, RB, MK, FXV), and the Neurochoice project of SystemsX.ch (KES), and the Hefter Research Center Zurich (FXV). We thank Erich Studerus and George Greer for critical comments on the manuscript and Marta Garrido for providing an example script of the roving MMN paradigm. We also acknowledge Milena Jeker for her assistance in recruiting and measuring.

\section{DISCLOSURE}

The authors declare no conflict of interest.

\section{REFERENCES}

Adler C, Malhotra A, Elman I, Goldberg T, Egan M, Pickar D et al (1999). Comparison of ketamine-induced thought disorder in healthy volunteers and thought disorder in schizophrenia. Am J Psychiatry 156: 1646-1649.

Alho K, Tervaniemi M, Huotilainen M, Lavikainen J, Tiitinen H, Ilmoniemi R et al (1996). Processing of complex sounds in the human auditory cortex as revealed by magnetic brain responses. Psychophysiology 33: 369-375.

Baldeweg T (2006). Repetition effects to sounds: evidence for predictive coding in the auditory system. Trends Cogn Sci 10: 93-94.

Baldeweg T, Klugman A, Gruzelier J, Hirsch S (2004). Mismatch negativity potentials and cognitive impairment in schizophrenia. Schizophr Res 69: 203-217.

Bodatsch M, Ruhrmann S, Wagner M, Müller R, Schultze-Lutter F, Frommann I et al (2010). Prediction of psychosis by mismatch negativity. Biol Psychiatry 69: 959-966.

Boly M, Garrido MI, Gosseries O, Bruno MA, Boveroux P, Schnakers C et al (2011). Preserved feedforward but impaired top-down processes in the vegetative state. Science 332: 858-862.

Braff DL, Geyer MA, Swerdlow NR (2001). Human studies of prepulse inhibition of startle: normal subjects, patient groups, and pharmacological studies. Psychopharmacology (Berl) 156: 234-258.

Brown AS (2011). The environment and susceptibility to schizophrenia. Prog Neurobiol 93: 23-58.

Chapman J (1966). The early symptoms of schizophrenia. Br J Psychiatry 112: 225-251.

Corlett PR, Frith CD, Fletcher PC (2009). From drugs to deprivation: a Bayesian framework for understanding models of psychosis. Psychopharmacology (Berl) 206: 515-530.

Corlett PR, Honey GD, Aitken MR, Dickinson A, Shanks DR, Absalom AR et al (2006). Frontal responses during learning predict vulnerability to the psychotogenic effects of ketamine: linking cognition, brain activity, and psychosis. Arch Gen Psychiatry 63: 611-621.

Corlett PR, Honey GD, Krystal JH, Fletcher PC (2011). Glutamatergic model psychoses: prediction error, learning, and inference. Neuropsychopharmacology 36: 294-315.

Corlett PR, Murray GK, Honey GD, Aitken MR, Shanks DR, Robbins TW et al (2007). Disrupted prediction-error signal in psychosis: evidence for an associative account of delusions. Brain 130: 2387-2400.

Cowan N, Winkler I, Teder W, Näätänen R (1993). Memory prerequisites of mismatch negativity in the auditory event-related potential (ERP). J Exp Psychol Learn Mem Cogn 19: 909-921.

Derogatis L (1994). SCL-90-R: Symptom Checklist-90-R Administration, Scoring and Procedures Manual. National Computer Systems: Minneapolis, MN.

Dittrich A (1975). Zusammenstellung eines Fragebogens (APZ) zur Erfassung abnormer psychischer Zustände [Construction of a questionnaire (APZ) for assessing abnormal mental states]. $Z$ Klin Psychol Psychiatr Psychother 23: 12-20.

Dittrich A (1998). The standardized psychometric assessment of altered states of consciousness (ASCs) in humans. Pharmacopsychiatry 31: 80-84.

Doeller CF, Opitz B, Mecklinger A, Krick C, Reith W, Schröger E (2003). Prefrontal cortex involvement in preattentive auditory deviance detection: neuroimaging and electrophysiological evidence. NeuroImage 20: 1270-1282.

Fantegrossi WE, Murnane KS, Reissig CJ (2008). The behavioral pharmacology of hallucinogens. Biochem Pharmacol 75: 17-33.

Fletcher PC, Frith CD (2009). Perceiving is believing: a Bayesian approach to explaining the positive symptoms of schizophrenia. Nat Rev Neurosci 10: 48-58.

Friston K (2005). A theory of cortical responses. Philos Trans R Soc Lond Ser B 360: 815-836.

Garrido M, Friston K, Kiebel S, Stephan K, Baldeweg T, Kilner J (2008). The functional anatomy of the MMN: a DCM study of the roving paradigm. NeuroImage 42: 936-944.

Garrido M, Kilner J, Kiebel S, Friston K (2009a). Dynamic causal modeling of the response to frequency deviants. J Neurophysiol 101: 2620-2631.

Garrido M, Kilner J, Stephan K, Friston K (2009b). The mismatch negativity: a review of underlying mechanisms. Clin Neurophysiol 120: 453-463.

Geyer MA, Vollenweider FX (2008). Serotonin research: contributions to understanding psychoses. Trends Pharmacol Sci 29: 445-453.

Giard MH, Perrin F, Pernier J, Bouchet P (1990). Brain generators implicated in the processing of auditory stimulus deviance: a topographic event-related potential study. Psychophysiology 27: 627-640.

González-Maeso J, Sealfon S (2009). Psychedelics and schizophrenia. Trends Neurosci 32: 225-232. 
Gouzoulis-Mayfrank E, Habermeyer E, Hermle L, Steinmeyer A, Kunert H, Sass H (1998). Hallucinogenic drug induced states resemble acute endogenous psychoses: results of an empirical study. Eur Psychiatry 13: 399-406.

Gray JA, Feldon J, Rawlins JNP, Smith AD (1991). The neuropsychology of schizophrenia. Behav Brain Sci 18: 617-680.

Haenschel C, Vernon D, Dwivedi P, Gruzelier J, Baldeweg T (2005). Event-related brain potential correlates of human auditory sensory memory-trace formation. J Neurosci 25: 10494-10501.

Harvey JA (2003). Role of the serotonin 5-HT(2A) receptor in learning. Learn Mem 10: 355-362.

Heekeren K, Daumann J, Neukirch A, Stock C, Kawohl W, Norra C et al (2008). Mismatch negativity generation in the human 5HT2A agonist and NMDA antagonist model of psychosis. Psychopharmacology (Berl) 199: 77-88.

Hemsley DR (1993). A simple (or simplistic?) cognitive model for schizophrenia. Behav Res Ther 31: 633-645.

Honey GD, Corlett PR, Absalom AR, Lee M, Pomarol-Clotet E, Murray GK et al (2008). Individual differences in psychotic effects of ketamine are predicted by brain function measured under placebo. J Neurosci 28: 6295-6303.

Imada T, Hari R, Loveless N, McEvoy L, Sams M (1993). Determinants of the auditory mismatch response. Electroencephalogr Clin Neurophysiol 87: 144-153.

Jääskeläinen IP, Ahveninen J, Bonmassar G, Dale AM, Ilmoniemi RJ, Levänen S et al (2004). Human posterior auditory cortex gates novel sounds to consciousness. Proc Natl Acad Sci USA 101: 6809-6814.

Javitt DC, Zukin SR (1991). Recent advances in the phencyclidine model of schizophrenia. Am J Psychiatry 148: 1301-1308.

Kandel ER (2001). The molecular biology of memory storage: a dialog between genes and synapses. Biosci Rep 21: 565-611.

Kantrowitz JT, Javitt DC (2010). Thinking glutamatergically: changing concepts of schizophrenia based upon changing neurochemical models. Clin Schizophr Relat Psychoses 4: $189-200$.

Kapur S (2003). Psychosis as a state of aberrant salience: a framework linking biology, phenomenology, and pharmacology in schizophrenia. Am J Psychiatry 160: 13-23.

Kapur S, Seeman P (2001). Ketamine has equal affinity for NMDA receptors and the high-affinity state of the dopamine D2 receptor. Biol Psychiatry 49: 954-957.

King R, Barchas JD, Huberman BA (1984). Chaotic behavior in dopamine neurodynamics. Proc Natl Acad Sci USA 81: 1244-1247.

Kometer M, Cahn BR, Andel D, Carter OL, Vollenweider FX (2011). The 5-HT2A/1A agonist psilocybin disrupts modal object completion associated with visual hallucinations. Biol Psychiatry 69: 399-406.

Korostenskaja M, iæ D, Kähkönen S (2008). The effect of methylphenidate on auditory information processing in healthy volunteers: a combined EEG/MEG study. Psychopharmacology (Berl) 197: 475-486.

Krüger-Thiemer E (1968). Continuous intravenous infusion and multicompartment accumulation. Eur J Pharmacol 4: 317-324.

Krystal JH, D’Souza DC, Mathalon D, Perry E, Belger A, Hoffman R (2003). NMDA receptor antagonist effects, cortical glutamatergic function, and schizophrenia: toward a paradigm shift in medication development. Psychopharmacology (Berl) 169: 215-233.

Lahti AC, Holcomb HH, Medoff DR, Tamminga CA (1995a). Ketamine activates psychosis and alters limbic blood flow in schizophrenia. NeuroReport 6: 869-872.

Lahti AC, Koffel B, LaPorte D, Tamminga CA (1995b). Subanesthetic doses of ketamine stimulate psychosis in schizophrenia. Neuropsychopharmacology 13: 9-19.
Leitman DI, Sehatpour P, Higgins BA, Foxe JJ, Silipo G, Javitt DC (2010). Sensory deficits and distributed hierarchical dysfunction in schizophrenia. Am J Psychiatry 167: 818-827.

Leung S, Croft RJ, Baldeweg T, Nathan PJ (2007). Acute dopamine $\mathrm{D}(1)$ and $\mathrm{D}(2)$ receptor stimulation does not modulate mismatch negativity (MMN) in healthy human subjects. Psychopharmacology (Berl) 194: 443-451.

Leung S, Croft RJ, Guille V, Scholes K, O’Neill BV, Phan KL et al (2010). Acute dopamine and/or serotonin depletion does not modulate mismatch negativity (MMN) in healthy human participants. Psychopharmacology (Berl) 208: 233-244.

Macmillan N, Creelman CD (1991). Detection Theory: A User's Guide. Cambridge University Press: Cambridge, MA.

Malhotra AK, Pinals DA, Weingartner H, Sirocco K, Missar CD, Pickar D et al (1996). NMDA receptor function and human cognition: the effects of ketamine in healthy volunteers. Neuropsychopharmacology 14: 301-307.

Moghaddam B, Adams B, Verma A, Daly D (1997). Activation of glutamatergic neurotransmission by ketamine: a novel step in the pathway from NMDA receptor blockade to dopaminergic and cognitive disruptions associated with the prefrontal cortex. J Neurosci 17: 2921-2927.

Morris RG, Anderson E, Lynch GS, Baudry M (1986). Selective impairment of learning and blockade of long-term potentiation by an $N$-methyl-D-aspartate receptor antagonist, AP5. Nature 319: 774-776.

Murray GK, Corlett PR, Clark L, Pessiglione M, Blackwell AD, Honey G et al (2008). Substantia nigra/ventral tegmental reward prediction error disruption in psychosis. Mol Psychiatry 13: 239, 267-276.

Muschamp JW, Regina MJ, Hull EM, Winter JC, Rabin RA (2004). Lysergic acid diethylamide and [-]-2,5-dimethoxy-4-methylamphetamine increase extracellular glutamate in rat prefrontal cortex. Brain Res 1023: 134-140.

Näätänen R (1992). Attention and Brain Function. Erlbaum Associates: Hillsdale, NJ.

Näätänen R (2000). Mismatch negativity (MMN): perspectives for application. Int J Psychophysiol 37: 3-10.

Näätänen R, Gaillard AW, Mäntysalo S (1978). Early selectiveattention effect on evoked potential reinterpreted. Acta Psychol (Amst) 42: 313-329.

Näätänen R, Winkler I (1999). The concept of auditory stimulus representation in cognitive neuroscience. Psychol Bull 125: 826859.

Nichols DE (2004). Hallucinogens. Pharmacol Ther 101: 131-181.

Opitz B, Rinne T, Mecklinger A, von Cramon DY, Schröger E (2002). Differential contribution of frontal and temporal cortices to auditory change detection: fMRI and ERP results. NeuroImage 15: $167-174$.

Rao RP, Ballard DH (1999). Predictive coding in the visual cortex: a functional interpretation of some extra-classical receptive-field effects. Nat Neurosci 2: 79-87.

Rinne T, Alho K, Ilmoniemi RJ, Virtanen J, Näätänen R (2000). Separate time behaviors of the temporal and frontal mismatch negativity sources. NeuroImage 12: 14-19.

Roiser JP, Stephan KE, den Ouden HE, Barnes TR, Friston KJ, Joyce EM (2009). Do patients with schizophrenia exhibit aberrant salience? Psychol Med 39: 199-209.

Sato Y, Yabe H, Hiruma T, Sutoh T, Shinozaki N, Nashida T et al (2000). The effect of deviant stimulus probability on the human mismatch process. NeuroReport 11: 3703-3708.

Sato Y, Yabe H, Todd J, Michie P, Shinozaki N, Sutoh T et al (2003). Impairment in activation of a frontal attention-switch mechanism in schizophrenic patients. Biol Psychol 62: 49-63.

Schneider W, Eschman A, Zuccolotto A (2002). E-Prime Reference Guide. Psychology Software Tools: Pittsburgh, PA.

Shaner A (1999). Delusions, superstitious conditioning and chaotic dopamine neurodynamics. Med Hypotheses 52: 119-123. 
Sheehan D, Lecrubier Y, Sheehan K, Amorim P, Janavs J, Weiller E et al (1998). The Mini-International Neuropsychiatric Interview (M.I.N.I.): the development and validation of a structured diagnostic psychiatric interview for DSM-IV and ICD-10. J Clin Psychiatry 59: 22-33.

Shi WX, Zhang XX (2003). Dendritic glutamate-induced bursting in the prefrontal cortex: further characterization and effects of phencyclidine. J Pharmacol Exp Ther 305: 680-687.

Shin KS, Kim JS, Kang DH, Koh Y, Choi JS, O'Donnell BF et al (2009). Pre-attentive auditory processing in ultra-high-risk for schizophrenia with magnetoencephalography. Biol Psychiatry 65: 1071-1078.

Stephan K, Baldeweg T, Friston K (2006). Synaptic plasticity and dysconnection in schizophrenia. Biol Psychiatry 59: 929-939.

Stephan K, Friston K, Frith C (2009). Dysconnection in schizophrenia: from abnormal synaptic plasticity to failures of self-monitoring. Schizophr Bull 35: 509-527.

Studerus E, Gamma A, Vollenweider F (2010). Psychometric evaluation of the altered states of consciousness rating scale (OAV). PLoS One 5: e12412.

Tiitinen H, Alho K, Huotilainen M, Ilmoniemi R, Simola J, Näätänen R (1993). Tonotopic auditory cortex and the magnetoencephalographic (MEG) equivalent of the mismatch negativity. Psychophysiology 30: 537-540.

Todd J, Robinson J (2010). The use of conditional inference to reduce prediction error - a mismatch negativity (MMN) study. Neuropsychologia 48: 3009-3018.

Turetsky BI, Calkins ME, Light GA, Olincy A, Radant AD, Swerdlow NR (2007). Neurophysiological endophenotypes of schizophrenia: the viability of selected candidate measures. Schizophr Bull 33: 69-94.

Umbricht D, Koller R, Vollenweider F, Schmid L (2002). Mismatch negativity predicts psychotic experiences induced by NMDA receptor antagonist in healthy volunteers. Biol Psychiatry 51: 400-406.

Umbricht D, Krljes S (2005). Mismatch negativity in schizophrenia: a meta-analysis. Schizophr Res 76: 1-23.

Umbricht D, Schmid L, Koller R, Vollenweider F, Hell D, Javitt D (2000). Ketamine-induced deficits in auditory and visual context-dependent processing in healthy volunteers: implications for models of cognitive deficits in schizophrenia. Arch Gen Psychiatry 57: 1139-1147.
Umbricht D, Vollenweider F, Schmid L, Grübel C, Skrabo A, Huber T et al (2003). Effects of the 5-HT2A agonist psilocybin on mismatch negativity generation and AX-continuous performance task: implications for the neuropharmacology of cognitive deficits in schizophrenia. Neuropsychopharmacology 28: $170-181$.

Vollenweider F, Kometer M (2010). The neurobiology of psychedelic drugs: implications for the treatment of mood disorders. Nat Rev Neurosci 11: 642-651.

Vollenweider FX, Geyer MA (2001). A systems model of altered consciousness: integrating natural and drug-induced psychoses. Brain Res Bull 56: 495-507.

Vollenweider FX, Leenders KL, Oye I, Hell D, Angst J (1997a). Differential psychopathology and patterns of cerebral glucose utilisation produced by $(S)$ - and $(R)$-ketamine in healthy volunteers using positron emission tomography (PET). Eur Neuropsychopharmacol 7: 25-38.

Vollenweider FX, Leenders KL, Scharfetter C, Maguire P, Stadelmann O, Angst J (1997b). Positron emission tomography and fluorodeoxyglucose studies of metabolic hyperfrontality and psychopathology in the psilocybin model of psychosis. Neuropsychopharmacology 16: 357-372.

Vollenweider FX, Vontobel P, Oye I, Hell D, Leenders KL (2000). Effects of $(S)$-ketamine on striatal dopamine: a $\left[{ }^{11} \mathrm{C}\right]$ raclopride PET study of a model psychosis in humans. J Psychiatr Res 34: 35-43.

Waberski T, Kreitschmann-Andermahr I, Kawohl W, Darvas F, Ryang Y, Gobbelé R et al (2001). Spatio-temporal source imaging reveals subcomponents of the human auditory mismatch negativity in the cingulum and right inferior temporal gyrus. Neurosci Lett 308: 107-110.

Williams GV, Rao SG, Goldman-Rakic PS (2002). The physiological role of 5-HT2A receptors in working memory. J Neurosci 22: 2843-2854.

Winkler I, Karmos G, Näätänen R (1996). Adaptive modeling of the unattended acoustic environment reflected in the mismatch negativity event-related potential. Brain Res 742: 239-252.

Wittchen HU, Pfister H (1997). DIA-X-Interviews: Manual für Screening-Verfahren und Interview. Swets Test Services: Frankfurt. 